\title{
PIV Investigation of the Flow Characteristics in an Internal Coolant Passage with Two Ducts Connected by a Sharp $180^{\circ}$ Bend
}

\author{
J. Schabacker, A. Bölcs \\ Swiss Federal Institute of Technology \\ EPFL-LTT \\ Lausanne, Switzerland
}

\author{
B.V. Johnson \\ ABB Corporate Research \\ Baden, Switzerland
}

\begin{abstract}
A PIV study of the flow in a stationary model of a smooth two-pass internal coolant passage is presented, which focuses on the flow characteristics in the sharp $180^{\circ}$ bend and downstream of the bend where the flow is redeveloping. Because PIV in its traditional conception only allows for the investigation of two-dimensional flow, a stereoscopic digital PIV system was assembled that measures all three velocity components simultaneously. The mean-velocity field and turbulence quantities of the flow are obtained from the PIV measurements.

The model of the coolant passage consists of two square ducts, each having a length of 19 hydraulic diameters, which are connected by a sharp $180^{\circ}$ bend with a rectangular outer wall. The measurements were obtained at one flow condition with a flow Reynolds number of 50,000 . A strong secondary flow motion occurs in the bend of the smooth passage that consists of two counter rotating vortices, which impinge on the outer wall and influence the flow in the downstream leg. Both of the outer corners contain regions of recirculating flow. A separation bubble, about 1.5 hydraulic diameters long, occurs on the inner wall of the return pass. In the symmetry plane, the flow recovers from the bend effect within 10 hydraulic diameters.
\end{abstract}

\section{Nomenclature}

Cartesian coordinate in axial duct direction

Cartesian coordinate in cross duct direction Cartesian coordinate in horizontal duct direction Cylindrical coordinate in streamwise direction Cylindrical coordinate in radial direction Mean velocity component in $\mathrm{x}$ direction Mean velocity component in y direction Mean velocity component in $\mathrm{z}$ direction Mean velocity component in streamwise direction in bend Mean velocity component in radial direction in bend Fluctuating velocity component in axial direction Fluctuating velocity component in cross duct direction w' Fluctuating velocity component in vertical direction $\mathrm{u}_{\phi}^{\prime} \quad$ Fluctuating velocity component in streamwise direction $\mathrm{u}_{\mathrm{r}}^{\prime} \quad$ Fluctuating velocity component in radial direction u'v',u'w' Cartesian components of turbulent shear stress $\mathrm{U}_{\mathrm{b}} \quad$ Bulk mean-velocity

D Height and width of passage legs, $\mathrm{D}=100 \mathrm{~mm}$

$\mathrm{D}_{\mathrm{h}} \quad$ Hydraulic diameter $\mathrm{D}_{\mathrm{h}}=\mathrm{D}$

B Thickness of divider plate

$\mathrm{S} \quad$ Section length in bend at $90^{\circ}$ section, $\mathrm{S} / \mathrm{D}_{\mathrm{h}}=1.05$

$\alpha \quad$ Section angle in bend

$\mathrm{k} \quad$ Turbulent kinetic energy $\frac{\frac{1}{2}\left(\left(\mathrm{u}^{\prime 2}+\mathrm{v}^{\prime 2}+\mathrm{w}^{\prime 2}\right)\right)}{\mathrm{U}_{\mathrm{b}}^{2}} \Leftrightarrow \frac{\frac{1}{2}\left(\left(\mathrm{u}_{\Phi}^{\prime 2}+\mathrm{u}_{\mathrm{r}}^{\prime 2}+\mathrm{w}^{\prime 2}\right)\right)}{\mathrm{U}_{\mathrm{b}}^{2}}$ in bend

proj Projected distance in bend $=$

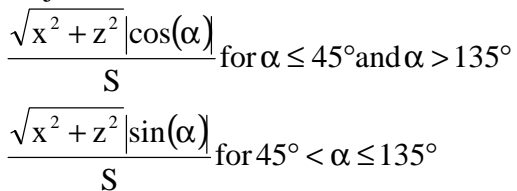

\section{Introduction}

Advanced gas turbines are designed to operate with high efficiency to decrease fuel consumption as well as the pollution of the environment. From basic thermodynamic consideration, it can be derived that the efficiency of the gas turbine process cycle rises with higher turbine pressure ratios and more importantly with higher turbine inlet temperatures.

The maximum turbine inlet temperature, however, is limited by the availability of blade material that can withstand such high temperatures and stresses. During the last 50 years a considerable increase of the turbine inlet temperature has been achieved, e.g. in Lakshminarayana (1996). Modern materials can withstand temperatures of about $1200 \mathrm{~K}$. Present gas turbines operate at turbine inlet temperatures of approximately $1550 \mathrm{~K}$. Effective cooling is applied to the turbine components that are exposed to the hot stream. 
For this, a combination of internal and external cooling methods is typically employed to lower the mean blade temperature to allowable values.

\section{Internal cooling of turbine blades}

For the internal cooling of the blades, the coolant is passed through a multipass circuit from hub to tip and ejected at the trailing edge or the blade tip. The straight sections of these coolant passages are connected by $180^{\circ}$ bends. To enhance heat transfer, the walls are roughened by ribs or pin fins that lead to very complicated flow patterns in the passage.

In the sharp $180^{\circ}$ bends, a Dean-type secondary motion is established. The secondary flow is formed by the imbalance between the radial pressure gradient and the centrifugal force that acts on the fluid. Due to the streamline curvature, a radial pressure gradient develops across the bend section with a higher pressure near the outer radius surface. The pressure imbalance acts on the slowly moving fluid near the sidewalls of the bend and displaces it along the sidewalls from the outer to the inner radius wall. Near the symmetry plane, the turning of the high momentum fluid produces centrifugal forces that drive the flow radially outward towards the outer radius wall. Consequently, a secondary flow field with two counter-rotating vortices develops in the bends of stationary passages.

The effect of the bend on the heat transfer in smooth and ribbed stationary passages has been experimentally studied by Johnson and Launder. (1985), Metzger and Sahm (1986), Han et al. (1988), Han and Zhang (1991), and Ekkad and Han (1995). The secondary flows and flow separation at the tip of the divider plate influence the heat transfer in the bend. As the flow progresses through the bend of a smooth passage, the presence of the secondary flow causes higher heat transfer on the top and bottom wall as well as on the outer wall. This was attributed to the higher turbulence of the flow and impingement in conjunction with the transport of cold fluid from the bulk towards the walls. Downstream of the bend, high turbulence mixing by flow separation and reattachment on the divider plate leads to an additional increase of the heat transfer. In passages, where oblique ribs are mounted in the straight sections, the ribs create a secondary flow, which interacts with the curvature induced secondary flow in the bend. This leads to very complicated flow fields in the turning region.

For the design of gas turbine blades, a detailed knowledge of the physical phenomena in the passage is necessary. Although CFD simulations can provide a better understanding of these phenomena, the numerical heat transfer predictions are not yet sufficiently accurate for design purposes. This is especially true for the complicated heat transfer patterns in the turning region of stationary or rotating coolant passages with rib roughened walls. To improve the performance of the CFD codes, a validation of the predictions is necessary and detailed measurements of the flow structure in the passages are required for comparison.

Previous experiments to define the flow characteristics and to provide data for CFD evaluation have included LDV measurements in rotating and stationary two-pass passages by Cheah et al. (1994) and Iacovides et al. (1996). Liou and Chen (1997) recently performed LDV measurements of the developing flow through a smooth duct with a $180^{\circ}$ straight-corner turn. Tse and Steuber (1997) reported 3D LDV measurements in rotating square serpentine coolant passage with skewed ribs.

\section{PIV technique}

In the present study, the particle-image-velocimetry (PIV) method was employed for the investigation of the flow field in a model of a stationary two-pass coolant passage. PIV was chosen for such a study because of its high data acquisition rate and the good spatial resolution of the measured flow fields. These features are obtained because PIV performs full-flow field measurements in a sheet of light in the flow. A good selection of papers on PIV can be found in Grant (1994). The reader is referred to this reference for a more detailed discussion of the requirements for the setup of PIV experiments.

Few PIV studies of flows in turbomachinery have been reported to date. Bryanston-Cross et al. (1991) carried out experiments in a transonic turbine cascade. Tisserant and Breugelsmans (1995) performed rotor blade-to-blade velocity measurements and Gogineni et al. (1996) studied periodically forced flat plate film cooling.

The measurements of highly three-dimensional flow with a singlecamera PIV system could have substantial errors because the out-ofplane motion of a particles perpendicular to the light sheet plane produces a systematic measurement error that increases with increasing distance from the optical axis, e.g., Lourenco (1988). In principle, an error correction for the in-plane components is feasible if the out-of-plane component of the flow is known. Schabacker and Bölcs (1996) applied the correction to single-camera 3D PIV velocity measurements and showed that the effect of the out-of-plane motion can not be corrected completely, although the correction improves the measurement accuracy.

This measurement error can be avoided with a stereoscopic PIV setup. A comprehensive analysis of the translation method, photographic recording, PIV-like evaluation of the separate records, matching of the data for three-dimensional velocity vectors and an application to rotating disc flow has been given by Prasad and Adrian (1993). Westerweel and Nieuwstadt (1991) have reported performance tests on three-dimensional velocity measurements with a digital twocamera stereoscopic PIV system.

There are two possible stereoscopic PIV configurations with which to view the light sheet at an oblique angle. In the angular displacement method, the imaging systems point at the particle field such that their optical axis form the required stereo angles with the light sheet. However, the oblique orientation of the object plane results in a tilted plane so that a normal camera could have focusing problems. Therefore, the angle is often kept so small that the resulting defocusing is acceptable. In the translation method, the imaging systems are aligned to right angles to the light sheet. The common field of view is limited due to the axial distance required for sufficient accuracy. Nevertheless, translating the back planes of the cameras can increase the common field of view (Hinsch 1995).

For the present investigation, a stereoscopic digital PIV system that employs the angular displacement method and commercially available devices was assembled. No modifications to the cameras were made. The small amount of defocusing of the images towards the edges was found to have a minor influence and was tolerated. The system is capable of measuring simultaneously all three velocity components. Subsequently, an ensemble average of the velocity data in identical spatial windows is calculated leading to the mean and fluctuating velocity field.

\section{Objectives of the study}

The objectives of the present paper are:

- To demonstrate that the stereoscopic PIV technique is capable of obtaining detailed statistical data that can be used for the evaluation of turbulence models in complex three-dimensional flows.

- To obtain and present data for turbulent flow in a smooth internal coolant passage with a sharp $180^{\circ}$ bend as the first demonstration case. 


\section{Experimental Setup}

\section{Test Facility}

A sketch of the test section is shown in Figure 1. Air was chosen as the working medium because heat transfer experiments will be conducted in the same facility in the near future. A continuously running compressor supplies the air to the test rig. Upstream of the test-rig, the mass flow rate is measured by means of an orifice meter. The air enters the settling chamber with an inner diameter of $600 \mathrm{~mm}$ via a $150 \mathrm{~mm}$ tube and a conical entrance section with an angle of $30^{\circ}$. The settling chamber is equipped with a combination of perforated plates, honeycombs and meshes to reduce unsteadiness and swirl in the flow. For the PIV experiments, 1-3 $\mu \mathrm{m}$ oil droplets generated by a Polytec L2F-A-1000 Aerosol Generator are injected upstream of the settling chamber to guarantee a homogeneous seeding density in the test section.
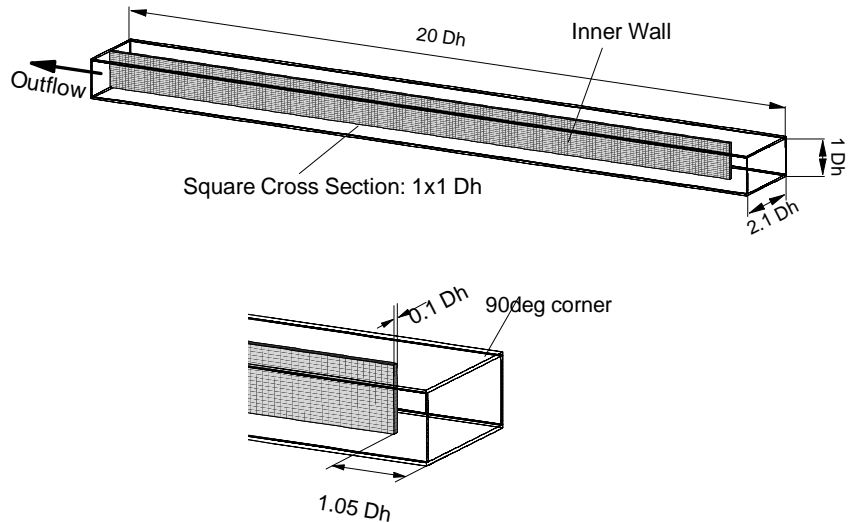

Figure 1 The internal coolant passage test facility

A modular concept was chosen for the test section design that allows an easy exchange of the components. Presently, the test rig is equipped with a model of a two-pass cooling passage of a gas turbine blade. The flow path in the downstream and upstream leg has a crosssection of 100x100 $\mathrm{mm}^{2}$ with a corresponding hydraulic diameter $\mathrm{D}_{\mathrm{h}}=100 \mathrm{~mm}$ and a length of $19 \mathrm{D}_{\mathrm{h}}$. The test section is made of 5-mm thick float glass to obtain good optical properties for the PIV experiment. In the straight-corner turn, the clearance between the tip of the divider plate and the outer wall is equal to $1.05 \mathrm{D}_{\mathrm{h}}$. The thickness of the divider plate is $0.1 \mathrm{D}_{\mathrm{h}}$.

The total section including the section entrance can be turned $90^{\circ}$ around the $\mathrm{x}$-axis without changing the flow conditions in the duct. This allows an easy optical access to the positions of interest for the PIV measurements.

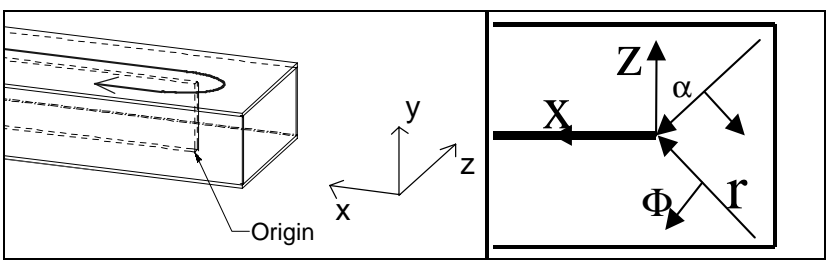

Figure 2 Definition of coordinate system. Left side: Cartesian system. Right side: Cylindrical system in bend

The definition of the coordinate systems in the test facility is shown in Figure 2. Two coordinate systems are used, a cartesian and a cylindrical. For both coordinate systems, the origin is set on the bottom wall at the turn entrance/exit plane. In the Cartesian $(\mathrm{x}, \mathrm{y}, \mathrm{z})$ system, $\mathrm{x}$ is defined as positive in the streamwise direction of the flow downstream of the bend exit, $y$ is defined positive vertically upwards in the horizontal test section orientation, and $\mathrm{z}$ is defined as shown.
In the cylindrical $(\Phi, \mathrm{r}, \mathrm{y})$ coordinate system, the radial component $\mathrm{r}$ is defined as positive in the direction towards the inner wall, the streamwise component $\Phi$ is defined as positive following the flow along the circular path centered on the center of the bend, and $y$ is defined as for the Cartesian system.

\section{The PIV system}

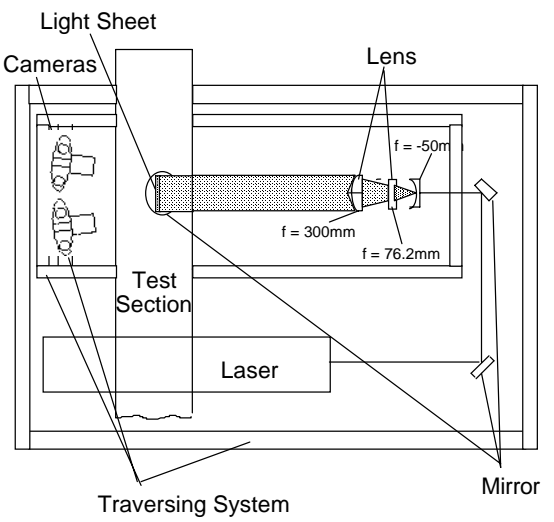

Figure 3 PIV setup

A Quantel TwinsB Nd-Yag double oscillator pulsed laser provides light pulses having a maximum energy of $320 \mathrm{~mJ}$ at a wavelength of $532 \mathrm{~nm}$. The time delay between a pair of pulses can be adjusted from $1 \mu \mathrm{s}$ to $1 \mathrm{~s}$ with a pulse duration of $5 \mathrm{~ns}$. A plano-concave lens $(-30 \mathrm{~mm}$ focal length) combined with two plano-cylindrical lenses (76.2 and $300 \mathrm{~mm}$ focal length) transform the beam into a thin vertical light sheet. By adjusting the distance between these lenses, the desired thickness and width of the light sheet can be obtained. All lenses have an anti-reflection coating. The complete system including laser, light sheet optics and camera is mounted on a traversing system shown in Figure 3 that allows an easy traverse to the position of interest.

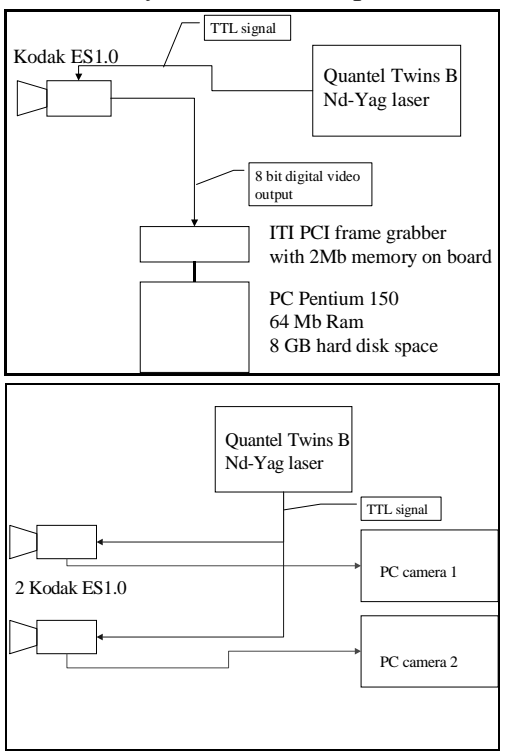

\section{Figure 4 Digital PIV system}

A sketch of the digital PIV system is shown in Figure 4. The system consists of two independent Kodak ES1.0 cameras each having its own PC. The ES1.0 has a CCD interline transfer sensor, which has a pixels array of $1008(\mathrm{H})$ by $1018(\mathrm{~V})$ pixels. Each pixel measures nine microns square with a $60 \%$ fill factor using a microlens. The camera outputs 8 bit digital images with 256 gray levels. For PIV applications, the camera is used in a special double exposure frame triggering mode. This mode allows the capture of two images 
separated by a delay ranging from $2 \mu$ s to $66 \mathrm{~ms}$. Nikkor $55 \mathrm{~mm}$ micro lenses are mounted on the cameras. For a typical recording situation, the cameras are placed with an oblique angle of $5^{\circ}$ at a distance of $0.7 \mathrm{~m}$ from the light sheet plane.

The PIV image acquisition starts with a signal from the laser. Two images are captured in rapid succession. This is accomplished by capturing the first image in the photo diode array, transferring this image to the CCD array and then capturing a second image in the photo diode array. The first image is transferred from the CCD to the frame grabber while the second image is being captured by the photo diode array. The second image is then transferred into the CCD array and subsequently onto the frame grabber's second image buffer. The frame grabber is an Imaging Technology PCI frame grabber with 2 MB memory onboard. The PCs are equipped with 64 MB RAM and 8 GB hard disk space. During the PIV measurement series, 50 images are written in real time into the PC's RAM memory. Subsequently, the acquisition is stopped and the images are saved on the hard disk.

\section{Data Reduction}

The PIV recordings from the right and left camera are interrogated independently with the PIV software package VISIFLOW from AEA Technology. For the images, a cross-correlation analysis method is used with an interrogation window size of 64 by 64 pixels and $50 \%$ overlap between the interrogation windows. The Kodak camera acquires images with a resolution of 1008 by 1018 pixels. Each recording results in a 30 by 30 vector field of the instantaneous velocity. Usually the data contains a small number of spurious vectors $(<2 \%)$. The vector field is therefore validated with predefined thresholds for the vector continuity and velocity magnitude. Vectors that do not fall within the thresholds are removed and the remaining gaps are filled by a weighted average of surrounding vectors.

From the processed vector fields, the instantaneous three-dimensional velocity field can be reconstructed. For angular PIV systems, where both cameras observe the light sheet from the same side, the corresponding interrogation positions for the two images in the plane of the light sheet do not match in general. Therefore, a calibration of the camera system is performed that also corrects for the distortion of the images in the lenses and the glass walls of the passage.

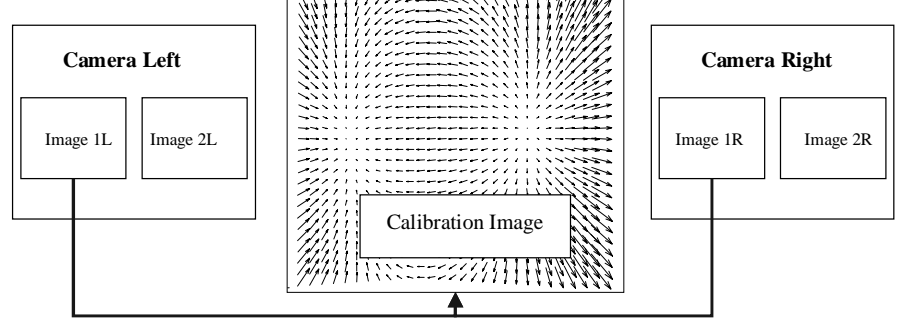

Figure 5 Calibration of the stereoscopic PIV system

A precision grid inserted into the field of view was used by others to find the matching points into the two images. In the present study, however, this is troublesome, since it requires opening the test section frequently. An alternative non-intrusive calibration technique was developed using the fact that both cameras record an identical particle pattern. For low particle densities, individual particles could be identified in corresponding images from the right and left camera and be used for the calibration of the camera arrangement. However, for typical particle densities in PIV images, it is difficult to find identical particles in both images. Therefore, groups of particles are correlated instead of tracking individual particles. Thus, a displacement field is processed from two corresponding images as shown in Figure 5.
The displacement vectors are arranged on a regular grid that is identical to the grid of the processed velocity vectors from the two cameras. In a subsequent step, the instantaneous three-dimensional velocity field can be reconstructed from the parallax (the apparent difference in the recorded displacements between the right and left image) between the images. For this, the velocity fields from the right and left camera are interpolated on an identical grid according to the allocation specification found from the calibration. For the interpolation, a linear algorithm is fitted to the measurements.

For more information on stereoscopic PIV imaging, the reader is referred to Westerweel and Nieuwstadt (1991). Note that the stereoscopic measurements provide the third velocity component, but even if the out-of-plane component is not required, stereoscopic recording increases the measurement accuracy because it corrects the in-plane velocity for the effects of perspective.

For most industrial applications, the engineers are interested not only in the instantaneous flow but also more importantly in the meanvelocity field and the turbulence quantities of the flow. In order to obtain PIV measurements in this form, the statistical distribution of the velocity components is calculated in identical spatial windows in a series of instantaneous PIV measurements. From these statistical distributions, the ensemble average and the statistical central moments are calculated which yield the desired mean-velocity field and Reynolds stresses.

\section{Applicability of the PIV technique to the presented investigation}

The previously presented PIV system provides velocity data at the location of the light sheet in the test section. All three velocity components and their turbulence quantities are measured simultaneously. The date acquisition rate and spatial resolution of the full-plane measurements is relatively high compared to conventional "point measurement" techniques as for example LDV. That is, because for a typical PIV experiment with the presented system, a matrix of $30 \times 30$ points is measured simultaneously with a point-wise data acquisition rate of approximately 2000 samples/hour. From the so obtained detailed vector map of the flow field, a good understanding of the 3D steady flow phenomena can be obtained particularly when the measurement plane is scanned through the flow volume. The data is arranged on a regular grid and is therefore ideally suited for the comparison with numerical flow simulations.

One sample, being a pair of PIV recordings, requires about $2 \mathrm{Mb}$ of disk space and thus limits the possible sample size for the calculation of mean velocities and turbulence quantities causing relatively high measurement uncertainty. For the mean velocity components, the measurement uncertainty can easily be decreased to lower levels if one concentrates on a single measurement plane in the flow field and increases the sample size there. However, for the PIV measurement of normal and shear stresses with an accuracy comparable to that obtained from single-point measurement techniques far more samples would be required that currently cannot be acquired with state-of-theart video and computer technique. Furthermore, the uncertainty in the stereoscopic measurements of the normal and shear stresses perpendicular to the light sheet plane is higher than that of the stresses within the light sheet plane due to the reconstruction of the velocity field. For this reason in the present study only the in-plane Reynolds stresses are presented. Another limitation of the PIV technique is the size of the measurement volume in the flow that is determined by the size of the interrogation window for the PIV image analysis. For the presented measurements, each vector represents the average of the flow within a volume of approximately $6 \times 6 \times 1 \mathrm{~mm}^{3}$, which is rather large. In principle, the size of the measurement volume could be decreased by increasing the magnification of the PIV recordings. For the angular stereoscopic system, the reduced depth of field of the PIV 
recording imposes a lower limit for the measurement volume as the distance between measurement-plane and image-plane of the cameras decreases. This effect is due to unfocused particles in the recordings that effect the measurement accuracy.

In the present study, therefore the measurements focus on the largescale turbulent interaction, which drive the overall flow field. The flow was investigated in various planes within the bend and downstream of the bend providing a good understanding of the large scale flow phenomena and with some restrictions also for the small scale phenomena in the bend corners shown in Figure 8, where the image magnification was increased. The authors believe that despite the limitation of PIV in terms of measurement accuracy and measurement volume, the results can be used for the validation of numerical codes. For the near wall characteristics of the flow it is recommended to combine the PIV technique with single-point measuring techniques.

\section{Measurements program and flow condition}

Detailed measurements of the flow structure in a smooth two-pass passage have been obtained upstream and downstream of the sharp $180^{\circ}$ bend and in the bend. The flow Reynolds number was 50,000.

As mentioned previously, the PIV system is mounted on a traversing unit that allows a flexible positioning of the light sheet plane in the position of interest. Typically, the PIV experiments were carried out in such a way that, at a constant streamwise position, measurements were taken in nine parallel planes, $0.1 \mathrm{D}_{\mathrm{h}}$ apart. The test section was then turned and the measurements were repeated yielding a grid of measurement planes in Figure 6 from which the three-dimensional velocity field can be obtained.

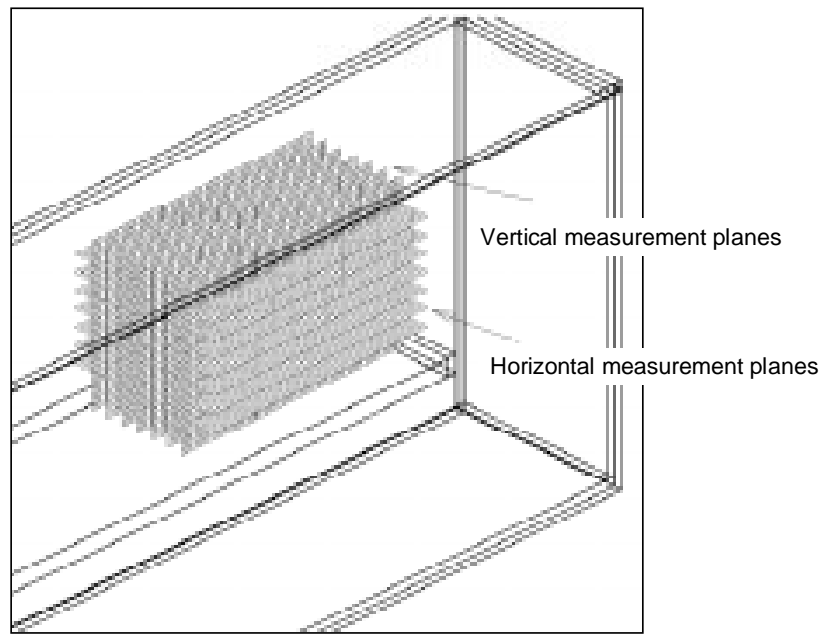

Figure 6 Combination of measurement planes

The vector maps that are presented in Figure 13 and Figure 15 were obtained from such a combination of measurement grids and thus have a more qualitative character. All other vector maps and profiles were extracted directly from the measurement planes and fall therefore within the uncertainty range specified in the following section.

\section{Uncertainty}

The estimation of the uncertainty of stereoscopic PIV velocity measurements requires the consideration of several aspects. Systematic errors occur due to the uncertainty in the determination of the geometrical parameter and the fabrication tolerances of the camera devices and lenses. Non-systematic errors are mainly due to the uncertainty in the determination of the average particle displacement in the interrogation region. The errors depend on the size of the interrogation region, the time separation between the laser pulses, the magnification of the recording, the out-of-plane velocity component, the turbulence of the flow, the length scale of the flow etc. The choice of the recording and interrogation parameters is therefore of significant importance for accurate and reliable velocity measurements.

To quantify the measurement uncertainty of the stereoscopic PIV system, an experimental study has been conducted. Typically, $100-$ 400 samples were ensemble averaged on each measurement plane. From these measurements, it was determined that for the presented study the $95 \%$ confidence interval for the mean-velocity field is less than $0.02 \mathrm{U}_{\mathrm{b}}$ in regions where the flow behaves well. Such a region is, for instance, the quasi two-dimensional flow in the bend symmetry plane as shown Figure 10. To quantify the uncertainty, the figure also includes the uncertainty interval for the mean velocities in Figure $10 \mathrm{a}+\mathrm{b}$. However, in regions of high turbulence and three-dimensional flow, as for example downstream of the bend exit in Figure 16a, the $95 \%$ confidence interval for the mean velocities increases to $0.10 \mathrm{U}_{\mathrm{b}}$. Due to the small sample size, the uncertainty interval for the corresponding turbulence quantities is rather high. The $95 \%$ uncertainty intervals for the fluctuating velocity components and Reynolds shear stresses were determined to be less than $0.05 \mathrm{U}_{\mathrm{b}}$ and $0.01 \mathrm{U}_{\mathrm{b}}^{2}$, respectively.

\section{Results and Discussion}

\section{Inlet conditions}
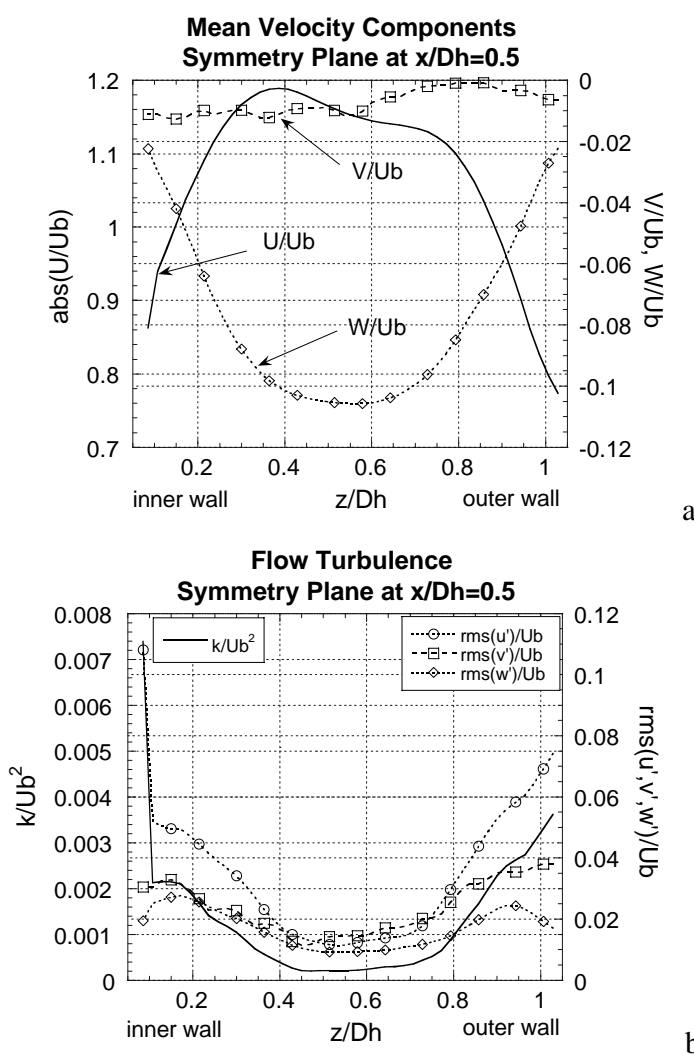

Figure 7 Inlet flow condition at $x / D_{h}=0.5$ upstream of bend

The flow condition at the bend inlet in the symmetry plane $\left(y=0.5 \mathrm{D}_{\mathrm{h}}\right)$ at $x / D_{h}=0.5$ is shown in Figure 7 . Near the divider wall $\left(\mathrm{z} / \mathrm{D}_{\mathrm{h}}=0.05\right)$, the bend causes a streamwise flow acceleration and consequently flow deceleration near the outer wall. The starting streamline curvature of the flow at this position is visible in the profile of the vertical velocity component which reaches a peak level of $\mathrm{W} / \mathrm{U}_{\mathrm{b}}=-0.105$ in the duct 
center. A slight asymmetry of the flow with respect to the symmetry plane can be observed in the profile of the cross-duct velocity component. The fluctuating velocity components shown in Figure $7 \mathrm{~b}$ reveal the isotropic turbulence of the flow in the duct center, whereas towards the wall the bend effect causes non-isotropic turbulence.

\section{Flow in bend region}

The flow in the symmetry plane $\left(y=0.5 \mathrm{D}_{\mathrm{h}}\right)$ of the bend region is shown in Figure 8. The figure was obtained from a superposition of different measurement position each having a square measuring area of 1 hydraulic diameter. Due to the streamline curvature of the flow in the bend, a radial pressure gradient develops across the bend section with higher pressure near the outer wall, Chang et al. (1983). As the flow approaches the bend, strong flow acceleration occurs near the inner wall due to the favourable pressure gradient there.
Flow deceleration takes place at the outer wall and is followed by flow separation, which results in a zone of recirculating flow in the upstream corner. The diameter of the separation bubble in the upstream corner of the symmetry plane is about $0.3 \mathrm{D}_{\mathrm{h}}$. A second, smaller bubble develops in the downstream corner where the high momentum flow and the higher pressure in the main stream keeps the size of the separation zone small.

As the flow progresses through the bend, secondary flows develop and the pressure field changes causing strong flow acceleration and impingement on the outer wall at the bend exit. Flow separation occurs in the bend entrance at the tip of the divider plate. Downstream of the bend exit a large separation exists that is adjacent to the inner wall. The flow reattaches in the symmetry plane on the inner wall at $\mathrm{x} / \mathrm{D}_{\mathrm{h}}=1.5-1.6$.
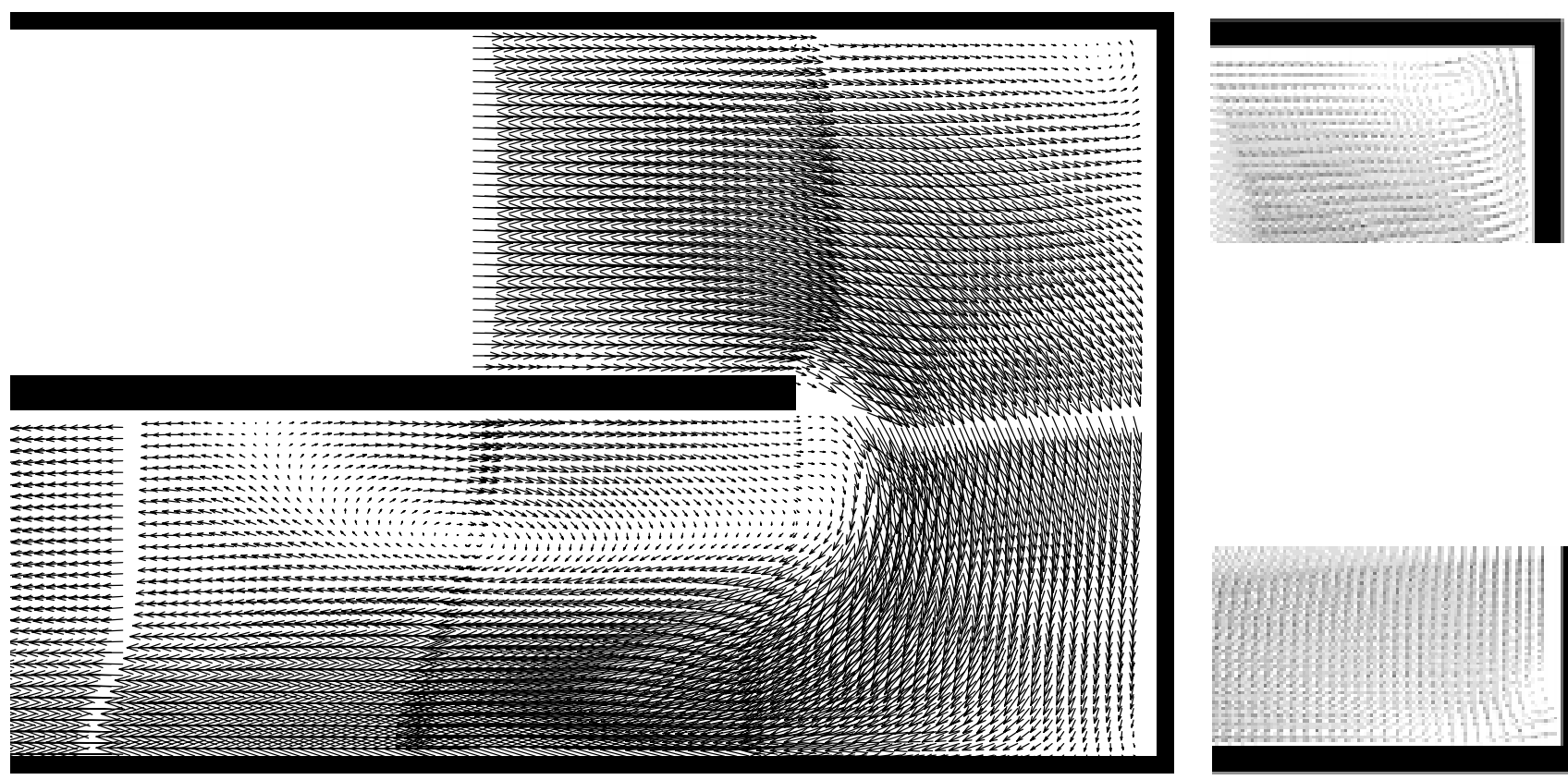

Figure 8 Flow field in the symmetry plane of bend region
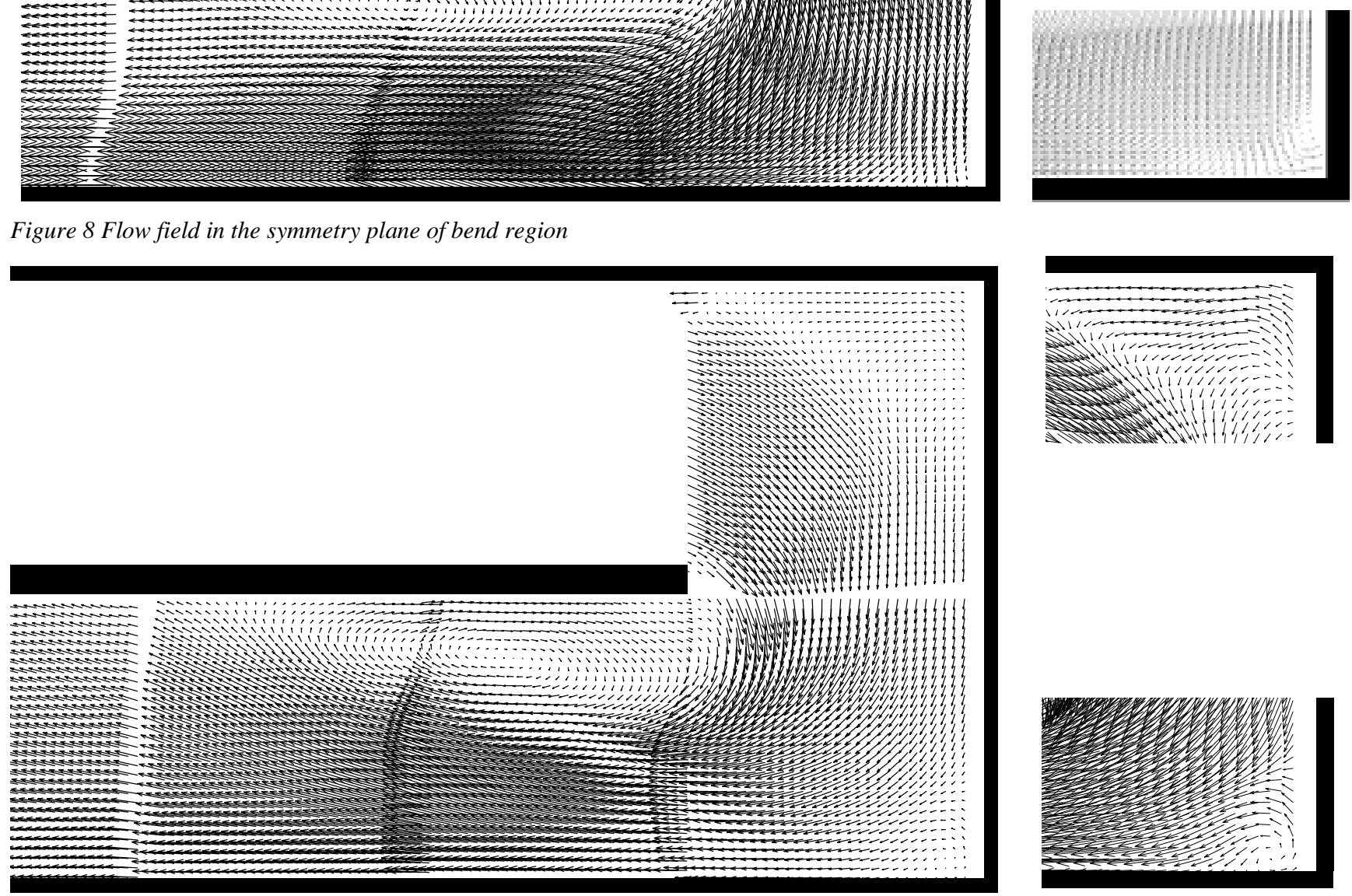

Figure 9 Flow in the $y / D_{h}=0.12$ plane of bend region

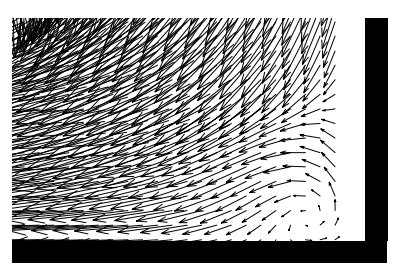



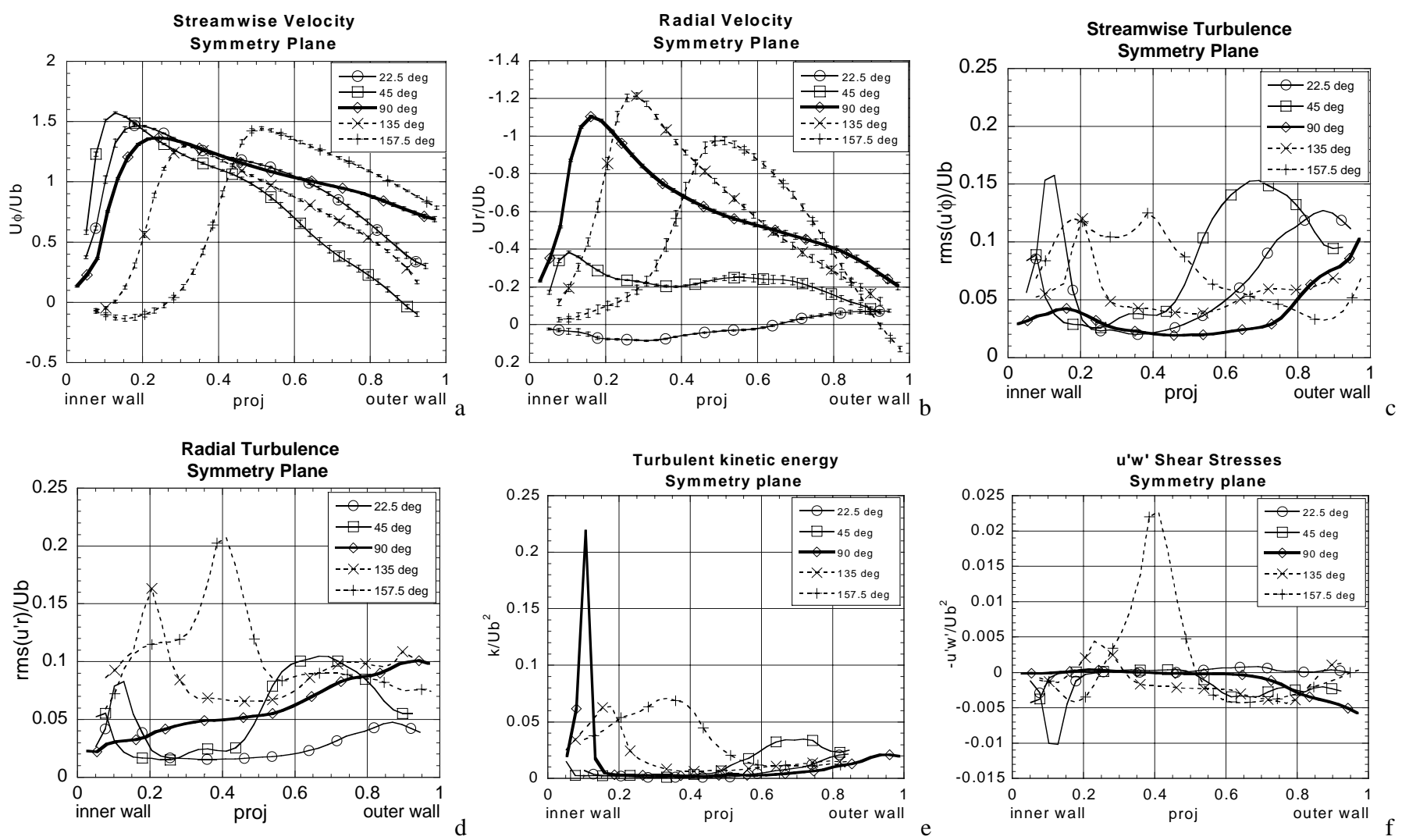

Figure 10 Flow development in the symmetry plane of the bend (including uncertainty intervals for mean velocities, Figure a+b)

The three-dimensional character of the flow is revealed in Figure 9, where the vector maps of the flow in a plane close to the bottom wall at $\mathrm{y} / \mathrm{D}_{\mathrm{h}}=0.12$ are presented.

In the $y / D_{h}=0.12$ plane, the combined effect of velocity defect on the bottom wall and secondary flow motion causes a larger vortex in the center of the separation bubble downstream of the bend exit. The center of the vortex is slightly shifted towards the inner wall. The reattachment length of the flow on the inner wall is increased by approximately 5\% with regards to the flow in the symmetry plane. The size of the recirculation zones in the outer upstream corner increases significantly towards the bottom wall, whereas in the downstream corner only a modest difference is observed. Because of the secondary flow that transports fluid in the bend towards the inner wall shown in Figure 13, the impingement of flow on the outer wall, which was observed in the symmetry plane, is decreased in the $\mathrm{y} / \mathrm{D}_{\mathrm{h}}=0.12$ plane.

A quantitative picture of the flow in the bend plane of symmetry is shown in Figure 10, where the development of the streamwise and radial velocity and the corresponding fluctuating velocity components are displayed. According to the previous discussion, the favorable pressure gradient causes the streamwise velocity $\mathrm{U}_{\Phi}$ to increase near the inner wall in the bend entrance shown in Figure 10a. As the flow progresses through the bend, the zone of high speed moves towards the outer wall, whereas flow with low velocity accumulates near the inner wall.

The radial velocity component shown Figure 10b, is directed everywhere towards the outer wall, except for the small region in the bend entrance $\left(22.5^{\circ}\right)$. Between $45^{\circ}$ and $90^{\circ}$, a strong development of secondary motion takes place that flows towards the outer wall with a speed of approximately $\mathrm{U}_{\mathrm{r}} / \mathrm{U}_{\mathrm{b}}=0.2$ at the $90^{\circ}$ section. Further downstream, the radial flow motion decreases owing to the streamwise flow acceleration in the outer radius half.

The destabilizing effect of the concave curvature at the outer wall and the recirculation zone in the upstream corner contribute to an increase of the flow turbulence in the outer radius half of the bend upstream of the $90^{\circ}$ cross-section shown in Figure $10 \mathrm{c}+\mathrm{d}$. The decrease of the streamwise fluctuating velocity component on the outer side over the second half of the bend is attributed to the strong streamwise flow acceleration. As the size of the separation bubble on the inner wall grows over the second half of the bend, the region of high turbulent kinetic energy shown in Figure 10e, expands from the inner side towards the outer region at $\alpha=135^{\circ}$ and $157.5^{\circ}$. The destabilising and stabilising curvature effects are believed to be responsible for producing anisotropic turbulence. The u'w' shear stresses in Figure 10f reveal the growing separation bubble on the inner wall at $\alpha=157.5^{\circ}$.

Near the bottom wall at $y / \mathrm{D}_{\mathrm{h}}=0.12$, the secondary flow vortex in Figure 13 causes high peak values in the cross-duct velocity shown in Figure $11 \mathrm{~b}$ and a strong radial velocity gradient in Figure 11a, at $\alpha=135^{\circ}$ and $\alpha=157.5^{\circ}$. The cross-duct velocity component transports fluid from the bottom into the bend center in the inner radius half and from the center towards the wall in the outer radius half, respectively. In the outer radius half, the radial velocity components contributes to a transport of fluid from the outer wall towards the bend circumferential mid-plane, whereas in the inner radius opposed fluid motion occurs. In particular at $\alpha=135^{\circ}$ and $\alpha=157.5^{\circ}$, shear stresses in Figure $11 \mathrm{c}$ occur that are attributed to the strong secondary flow motion in this region. 

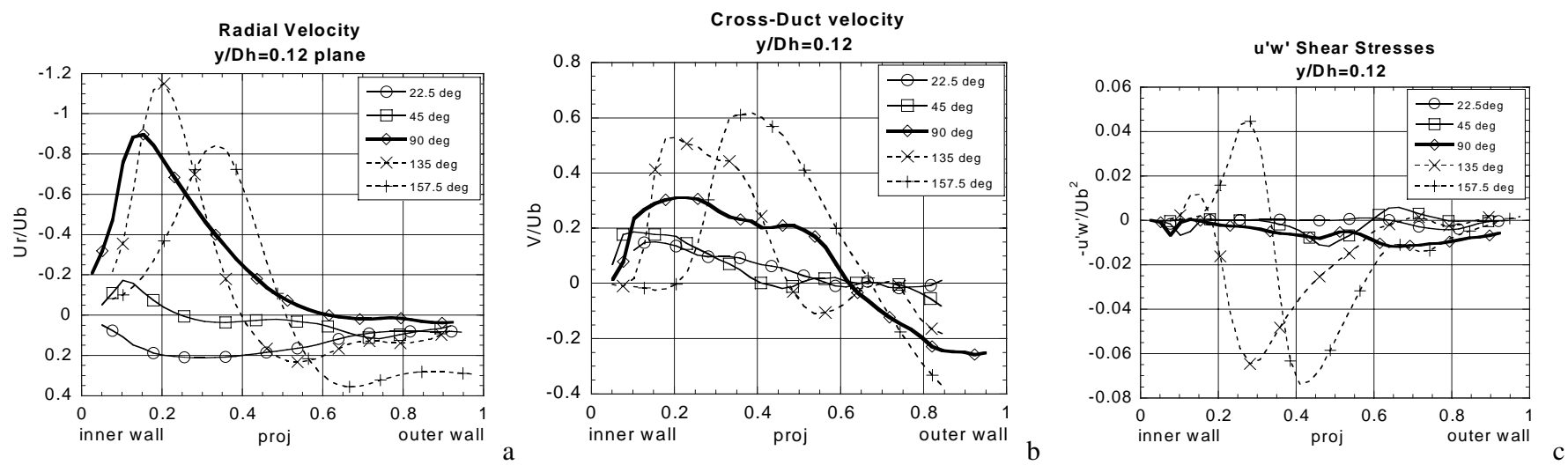

Figure 11 Flow development in the $y / D_{h}=0.12$ plane of the bend

In the bend, a Dean-type secondary motion establishes. The secondary flow fields in the $90^{\circ}$ and $157.5^{\circ}$ cross-sections in the bend are shown in Figure 13. Two counter-rotating vortices develop in the bend. In the symmetry plane, the vortices convect fluid towards the outer wall, causing a strong impingement of flow on the wall. Near the sidewalls, fluid is convected into the duct core. As the flow progresses through the bend, the centers of the two vortices occur further outwards which is compatable with the growing separation bubble on the inner wall. Simultaneously, the magnitude of the secondary flow motion increases in the circumferential mid-plane as shown in Figure 12, where mean velocities and turbulent kinetic energy are presented Almost flat radial velocity profiles shown in Figure $12 \mathrm{~b}$ are measured for $0.3<y / D_{h}<0.7$. Towards the top and bottom wall, a velocity defect up to $30 \%$ of the duct height occurs. As the flow progresses through the bend along the circumferential mid-plane in Figure 12a, the streamwise velocity decelerates from $\alpha=39^{\circ}$ to $\alpha=90^{\circ}$, followed by acceleration from $\alpha=113^{\circ}$ to $\alpha=121^{\circ}$.
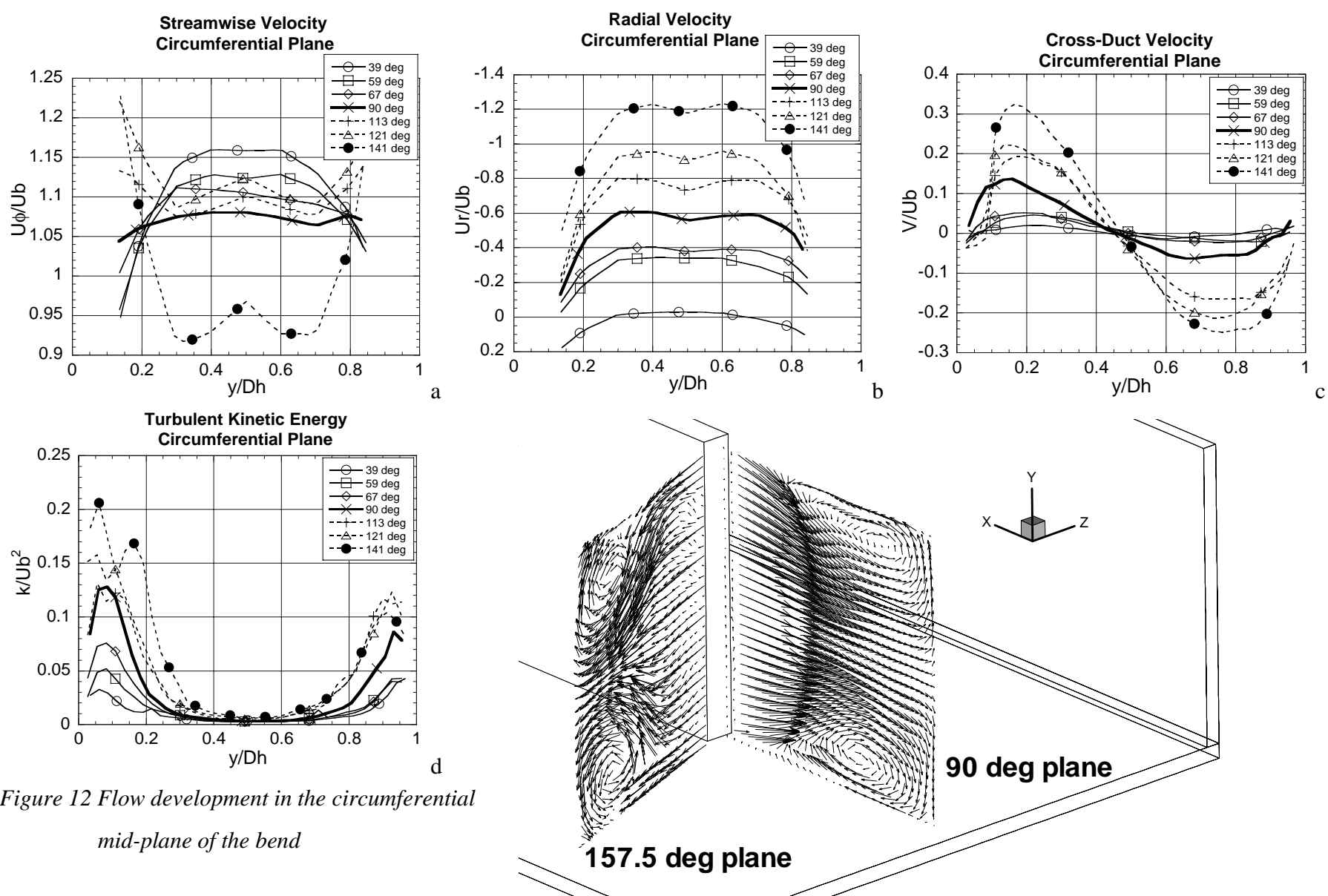

Figure 13 Secondary flow for $90 \mathrm{deg}$ and $157.5 \mathrm{deg}$ cross-sections of the bend 
A strong deceleration of the streamwise component occurs in the last quarter of the bend. At $\alpha=141^{\circ}$ the streamwise velocity has a decrease in the velocity near the central part of the duct. The decrease is related to the secondary flow motion that, at this position, transports fluid from the sidewalls towards the bend core. The radial velocity component shown in Figure 12b, increases from approximately zero velocity at $\alpha=39^{\circ}$ up to 1.2 times the downstream bulk velocity at the $\alpha=141^{\circ}$ cross-section.

The cross-duct velocity profiles in Figure 12c, show the developing secondary flow motion in the bend. Note that modest asymmetry occurs in the mean-velocity and turbulent kinetic energy profiles. This is attributed to small variations in the flow upstream of the bend and the unstable nature of the flow in the bend region. Most of the turbulent kinetic energy in Figure $12 \mathrm{~d}$ is produced near the walls where high velocity gradients and shear stresses act on the flow.

\section{Flow downstream of the bend exit}

The vector map of the secondary flow in cross-sections downstream of the bend exit is shown Figure 15. The two counter-rotating vortices, which are created in the bend, enter the downstream leg and vanish rapidly as the flow progresses further downstream. On the inner wall, a three-dimensional separation bubble can be observed.
The secondary flow transports fluid in the symmetry plane towards the outer wall and back to inner wall along the sidewalls.

A quantitative representation of the flow in the downstream leg is given in Figure 14, where the development of the flow quantities in the symmetry plane is displayed.

Near the bend exit at $\mathrm{x} / \mathrm{D}_{\mathrm{h}}=0.25$, the separation bubble in the symmetry plane has grown to approximately $40 \%$ of the duct height and increases to a maximum size of $45 \%$ at the $\mathrm{x} / \mathrm{D}_{\mathrm{h}}=0.75$ section. The flow undergoes a strong acceleration at the outer wall because of the secondary flow that transports fluid into this region. A comparison with Figure 10 shows that the streamwise velocity component increases from $\mathrm{U}_{\Phi} / \mathrm{U}_{\mathrm{b}}=1.4$ at the $157.5^{\circ}$ cross-section to $\mathrm{U} / \mathrm{U}_{\mathrm{b}}=1.9$ at $\mathrm{x} / \mathrm{D}_{\mathrm{h}}=0.25$. The peak in the velocity is shifted towards the outer wall. The streamwise velocity reaches a maximum value of $\mathrm{U} / \mathrm{U}_{\mathrm{b}}=2.2$ at the $\mathrm{x} / \mathrm{D}_{\mathrm{h}}=0.75$ section. With increasing distance from the bend exit, the flow decelerates quite rapidly. At $\mathrm{x} / \mathrm{D}_{\mathrm{h}}=4.2$ a streamwise velocity component of $U / U_{b}=1.2$ is measured, whereas at $x / D_{h}=10$ the flow characteristics are approaching those upstream of the bend.
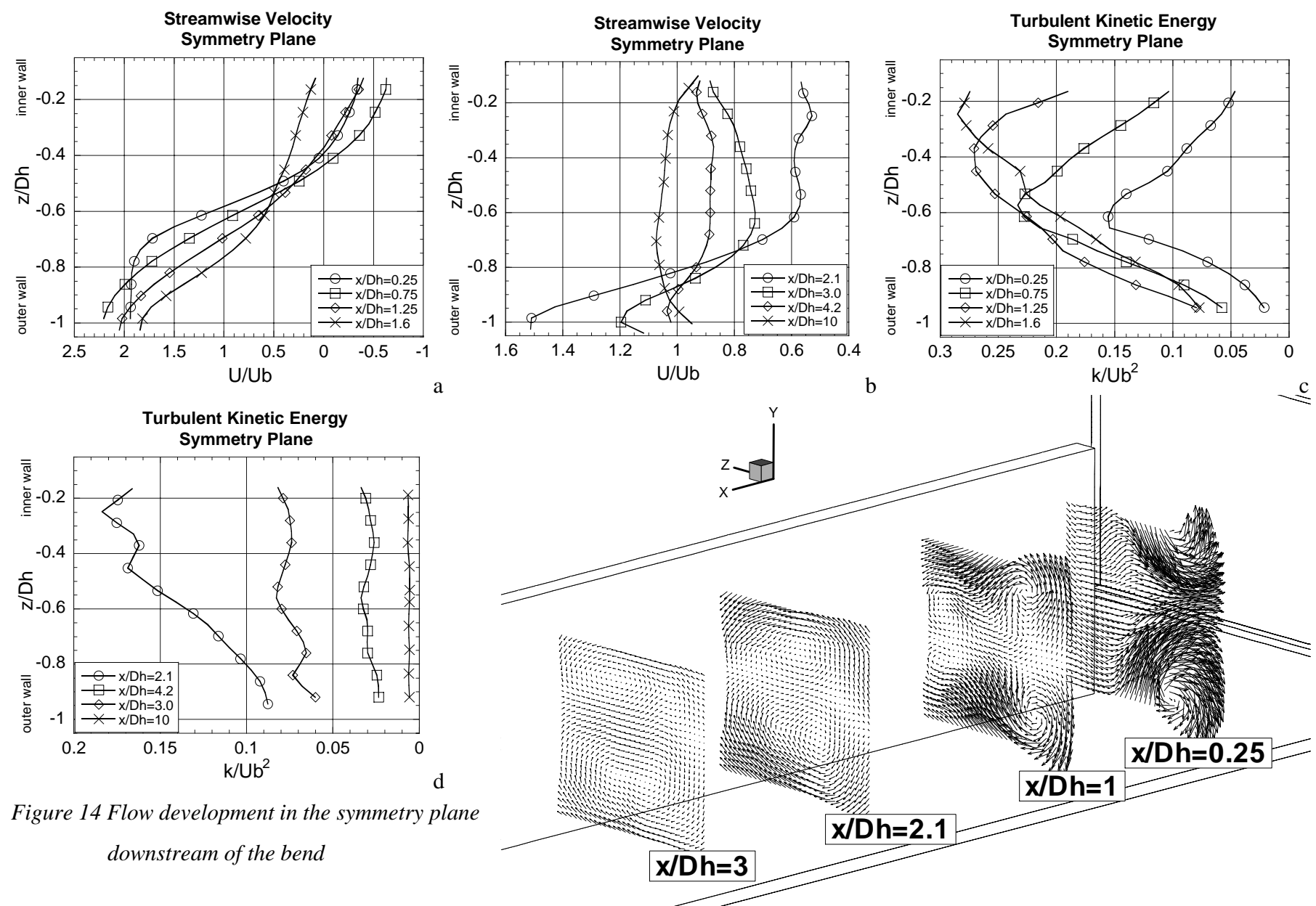

Figure 15 Secondary flow downstream of the bend

(The figure was obtained from the interpolation of a 9x9 grid as shown in Figure 6) 

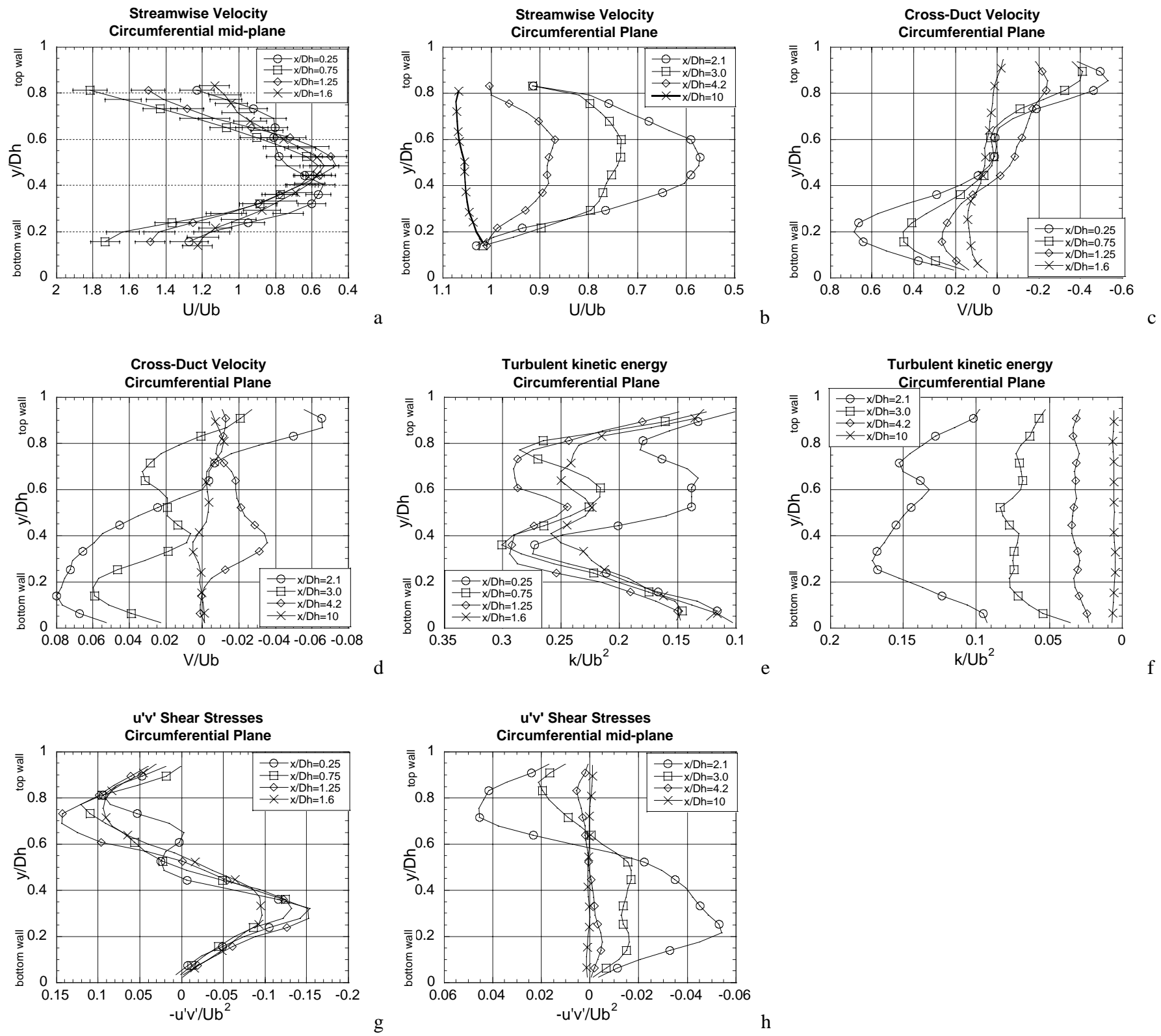

Figure 16 Flow development in circumferential mid-plane downstream of the bend (including uncertainty interval for streamwise mean-velocity, Figure 16a)

A similar trend is found at the inner wall. However, here the streamwise velocity is in the reversed direction upstream of the reattachment point. The maximum magnitude $\left(\mathrm{U} / \mathrm{U}_{\mathrm{b}}=0.6\right)$ of this reverse flow motion occurs at $\mathrm{x} / \mathrm{D}_{\mathrm{h}}=0.75$. The flow reattaches on the inner wall at $\mathrm{x} / \mathrm{D}_{\mathrm{h}}=1.5-1.6$. Hereafter the redevelopment of the boundary layer starts.

The peak values of the turbulent kinetic energy occur in the shear layer between the recirculation bubble and the main flow in a region where high velocity gradients and shear stresses cause high turbulent energy production. The highest turbulence levels of $\mathrm{k} / \mathrm{U}_{\mathrm{b}} 2 \sim 0.28$ are measured near the reattachment point. Further downstream, the turbulent kinetic energy dissipates gradually towards a low turbulence level.The developments of streamwise velocity, turbulent kinetic energy, and u'v' shear stresses in the circumferential mid-plane downstream of the bend are shown in Figure 16, respectively. They are included to complete the database. These flow characteristics are compatible with those shown in Figure 14. Note that the profiles at $\mathrm{x} / \mathrm{D}_{\mathrm{h}}=10$ in both the symmetry and circumferential plane are approximately equal.

\section{Conclusions}

In the present paper, a stereoscopic PIV system has been employed for the measurement of the turbulent flow characteristics in a model of a gas turbine coolant passage.

The measurements have been carried out in a passage with smooth walls and a sharp $180^{\circ}$ bend. The PIV technique was shown to be capable of obtaining velocity measurements with a high spatial resolution and a reasonably small uncertainty of the results. These measurements are especially useful in the highly turbulent separation bubble downstream of the bend, where conventional "point measurement" techniques have yielded limited resolution of the flow 
characteristics. The data acquisition rate was high and thus allowed for the investigation of the flow in a complex geometry where many measurement planes are required to obtain a comprehensive understanding of the flow phenomena and a large database to evaluate turbulence models for CFD codes. From the statistical distribution of the velocity components, the mean-velocity field and turbulent transport of the flow were calculated. The velocity fields were arranged on a regular grid and can therefore ideally be used to evaluate CFD simulations.

As expected, the PIV measurements showed that the flow in the coolant passage was characterized by the development of a strong Dean-type secondary flow in the bend. The secondary flow consisted of two counter rotating vortices that transport fluid in the bend center from the inner wall towards the outer. Close to the sidewalls, the secondary flow was convected to the inner wall. Downstream of the bend exit, a large separation bubble existed on the inner wall. In the symmetry plane, the flow reattached at 1.5-1.6 hydraulic diameters from the bend exit and towards the sidewalls the reattachment length increased. In both outer corners, zones of recirculating flow were measured. The secondary flow caused a strong impingement of the flow on the outer walls at the bend exit.

Because of the space limitations, only a fraction of the results that were obtained by the measurements could be presented in this paper. For a more complete database, the readers are referred to the senior author, J.S.

\section{Acknowledgement}

This study was supported by ABB Corporate Research, Ltd., Switzerland.

\section{References}

P.J. Bryanston-Cross, P.J. Towers, C.E. Judges, S.P. Harasgama, 1991, "The Application of Particle Image Velocimetry in a Short Duration Transonic Annular Turbine Cascade," ASME paper 91-GT221

S.M. Chang, J.A.C. Humphrey, A. Modavi, 1983, "Turbulent Flow in a Strongly Curved U-Bend and Downstream Tangent of Square Cross-Sections," PCH PhysicoChemical Hydrodynamics, Vol. 4, No. 3, pp. 243-269

S.C. Cheah, H. Iacovides, D.C. Jackson, H. Li, B.E. Launder, 1994", LDA Investigation of the Flow Development Through Rotating UDucts," ASME paper 94-GT-226

S.V. Ekkad, J.C. Han, 1995,'Local Heat Transfer Distribution Near a Sharp $180^{\circ}$ Turn of a Two-Pass Smooth Square Channel Using a Transient Liquid Crystal Image Technique," J. Flow Vis. Image Proc,. Vol. 2, pp. 285-297

S.P. Gogineni, D.D Trump, R.B. Rivir, D.J. Pestian, 1996, "PIV Measurements of Periodically Forced Flat Plate Film Cooling Flows with High Free Stream Turbulence," ASME paper 96-GT-236

Ian Grant (editor), 1994, "Selected Papers on Particle Image Velocimetry," Spie Milestone Series, Vol. MS 99

J.C. Han, P. Zhang, 1991, "Effect of Rib-Angle Orientation on Local Mass Transfer Distribution in a Three-Pass Rib-Roughened Channel," Journal of Turbomachinery, Vol. 113, pp. 123-130

J.C. Han, P.R. Chandra, S.C. Lau, 1988, "Local Heat/Mass Transfer Distributions Around Sharp $180^{\circ}$ Turns in Two-Pass Smooth and RibRoughened Channels," ASME Journal of Heat Transfer, Vol. 110, pp. 91-98

K.D Hinsch, 1995, “Three-Dimensional Particle Velocimetry,” Meas. Sci. Technol. Vol. 6, pp. 742-753

H. Iacovides, D.C. Jackson, H. Li, G. Kelemenis, B.E. Launder, K. Nikas, 1996, "LDA Study of the Flow Development through an
Orthogonally Rotating U-Bend of Strong Curvature and Rib Roughened Walls," ASME paper 96-GT-476

R.W. Johnson, B.E. Launder, 1985, "Local Heat Transfer Behaviour in Turbulent Flow Around a $180 \mathrm{deg}$ Bend of Square Cross Section," ASME paper 85-GT-68

B. Lakshminarayana, 1996, Fluid Dynamics and Heat Transfer of Turbomachinery, John Wiley \& Sons, Inc.

T.M. Liou, C.C. Chen, 1997, "LDV Study of Developing Flows through a Smooth Duct with a 180 deg Straight-Corner Turn," ASME paper 97-GT-283

L.M. Lourenco, 1988, "Some Comments on Particle Image Displacement Velocimetry," Von Karman Institute for Fluid Dynamics, Lecture Series 1988-06

D.E. Metzger, M.K. Sahm, 1986, "Heat Transfer Around Sharp $180^{\circ}$ Turns in Smooth Rectangular Channels," Journal of Heat Transfer, Vol. 108, pp. 500-506

A.K Prasad, R.J Adrian, 1993, "Stereoscopic Particle Image Velocimetry Applied to Liquid Flows," Experiments in Fluids, Vol. 15, pp. 49-60

J. Schabacker, A. Bölcs, 1996, "Investigation of Turbulent Flow by Means of the PIV Method," Paper presented at the $13^{\text {th }}$ Symposium on Measuring Techniques for Transonic and Supersonic Flows in Cascades and Turbomachines, Zurich, Switzerland, September 5-6

D. Tisserant, F.A.E. Breugelmans, 1995, "Rotor Blade-to-Blade Measurements Using Particle Image Velocimetry," ASME paper 95GT-99

D.G.N. Tse, G.D. Steuber, 1997, "Flow in a Rotating Square Serpentine Coolant Passage with Skewed Trips," ASME paper 97GT-529

J. Westerweel, F. T. Nieuwstadt, 1991, "Performance Tests on 3Dimensional Velocity Measurements with a Two-Camera Digital Particle-Image-Velocimeter," Laser Anemometry, Vol. 1, pp. 349355 